\begin{tabular}{|c|c|}
\hline Citation & $\begin{array}{l}\text { Annick Vogels, Sara Schevenels, Richard Cayenberghs, Eddy Weyts, } \\
\text { Griet Van Buggenhout, Ann Swillen, Hilde Van Esch, Thomy de Ravel, } \\
\text { Pieter Corveleyn, Koen Devriendt (2014), } \\
\text { Title Presenting symptoms in adults with the 22q11 deletion } \\
\text { syndrome } \\
\text { Eur. J. Med. Genet., Ahead of print. }\end{array}$ \\
\hline Archived version & $\begin{array}{l}\text { Author manuscript: the content is identical to the content of the } \\
\text { published paper, but without the final typesetting by the publisher }\end{array}$ \\
\hline Published version & $\begin{array}{l}\text { insert link to the published version of your paper } \\
\text { http://dx.doi.org/10.1016/j.ejmg.2014.02.008 }\end{array}$ \\
\hline Journal homepage & $\begin{array}{l}\text { insert link to the journal homepage of your paper } \\
\text { http://www.journals.elsevier.com/european-journal-of-medical- } \\
\text { genetics/. }\end{array}$ \\
\hline Author contact & $\begin{array}{l}\text { your email annick.vogels@uzleuven.be } \\
\text { Klik hier als u tekst wilt invoeren. }\end{array}$ \\
\hline IR & url in Lirias https://lirias.kuleuven.be/handle/123456789/445506 \\
\hline
\end{tabular}

(article begins on next page) 


\section{Presenting symptoms in adults with the 22q11 deletion syndrome}

Annick Vogels ${ }^{\mathrm{a} *}$, Sara Schevenels ${ }^{\mathrm{b}}$, Richard Cayenberghs ${ }^{\mathrm{c}}$, Eddy Weyts ${ }^{\mathrm{c}}$, Griet Van

Buggenhout $^{\mathrm{a}}$, Ann Swillen ${ }^{\mathrm{a}}$, Hilde Van Esch ${ }^{\mathrm{a}}$, Thomy de Ravel ${ }^{\mathrm{a}}$, Pieter Corveleyn ${ }^{\mathrm{b}}$, Koen

Devriendt $^{\mathrm{a}}$

${ }^{a}$ Center for Human Genetics, KU Leuven,

${ }^{b}$ Department of Psychiatry, University Hospitals, Leuven

${ }^{c}$ Sint-Kamillusziekenhuis, Bierbeek, Belgium

*Corresponding author. Tel.:+32 16346038

E-mail address: Annick.Vogels@uzleuven.be

Herestraat 49, 3000 Leuven, Belgium 


\section{ABSTRACT}

A definitive molecular diagnosis of 22q11 Deletion Syndrome (22q11DS) even if occurring later in life, has important genetic, medical and emotional impact on the patients and their families. The aim of this study is to describe presenting symptoms and age at diagnosis in an adult 22q11DS population

A retrospective study was performed on 65 individuals diagnosed with 22q11DS at adult age. Data were collected on adults referred to the genetic clinic or actively recruited through systematic diagnostic examination in both institutions and a psychiatric unit for intellectually disabled. Presenting symptoms were categorized into seven groups: familial occurrence, intellectual disability, cardiac anomalies, palatal anomalies, facial dysmorphic features, psychiatric problems and 'other' (comprising all other features associated with 22q11DS). Age at diagnosis was defined as the age at which the 22q11.2 deletion was detected by fluorescence in situ hybridization or comparative genomic hybridization. Ascertainment subgroups were different in presenting symptoms and age at diagnosis. Adults were referred to the genetic clinic mainly because of familial occurrence, cardiac defects and psychiatric disorders whereas adults diagnosed in institutions for intellectually disabled presented mainly with moderate to severe intellectual disability and psychotic disorders. Adults diagnosed at the psychiatric unit for intellectually disabled had a variety of psychiatric disorders but none of them had additional physical features.

This emphasizes the need to stay alert for presenting symptoms such as conotruncal heart defects or moderate to severe intellectual disability in combination with a history of psychiatric disorders, even in the absence of obvious physical features. 
Key-words:

22q11 deletion syndrome, presenting symptoms, age at diagnosis, intellectual disability, psychiatry, adults 


\section{Introduction}

The velocardiofacial syndrome also known as the 22q11.2 deletion syndrome (22q11DS), is the most common microdeletion syndrome with an estimated prevalence of one in 3000 to 6000 births [1]. The clinical phenotype is highly variable even within families [2] and more than 180 associated anomalies have been described [3].

McDonald-McGinn and Sullivan [1] proposed three categories in which to divide the possible phenotypic features according to their frequency and their contribution to the patient's quality of life. The most common phenotypic features contributing substantially to the medical needs of the patients are defined as major phenotypic features which include developmental delay, cardiac anomalies, palatal anomalies and immune deficiency. Intermediate phenotypic features are less common but medically important and comprise renal anomalies, hypocalcaemia, feeding problems and psychiatric disorders. Minor medical features, including the characteristic facial features, contribute to the overall clinical picture but are not a major concern to the patient's medical needs. None of these features occur in all patients and none are obligatory [3]. The clinical diagnosis is confirmed by a routine test based on FISH, available in most cytogenetic laboratories [2].

The initial diagnosis of 22q11DS in an individual is dependent on a number of factors, including the severity of the phenotypic features, the awareness of the physician and the patient's age [4].

Most patients are identified at a young age [5]. In infants, congenital cardiac anomalies [6, 7], cleft palate, feeding difficulties with nasal reflux, congenital thymic hypoplasia and neonatal hypocalcemia can lead to the diagnosis of 22q11DS. The majority of the children diagnosed after the age of two present with speech-language impairment, developmental delay or learning difficulties and recurrent infections [8]. The median age of diagnosis of children referred by pediatric cardiologists is 0.5 years, whereas the median age is 8 years for 
those referred by speech pathologists or cleft palate surgeons and 11.2 years for children referred by neurologists/psychiatrists [9].

Due to the variability of the phenotypic features and severity, the diagnosis may be missed in children [9] and molecular testing is still not performed in many instances where it may have been indicated [4]. These undiagnosed patients may not receive adequate social, medical and psychiatric care in adolescence and adulthood. A recent survey [10] reported additional important psychological benefits for the family of individuals diagnosed at a later age, such as having an explanation for a lifelong history of the medical, intellectual and behavioral problems, a better understanding of expectations and prognosis and a facilitation of access to special programs.

This study describes the age at diagnosis and the presenting symptoms in 22q11DS adults with the aim to identify adult patient groups in whom genetic testing is clinically warranted.

\section{Methodology}

\section{Sample}

"Between January first 1994 and December 31th, 2012, FISH analysis using a probe from the commonly deleted 22q11 region was performed in 1241 adults (minimum age 18 years) at the Centre for Clinical Genetics of the University Hospitals of Leuven. Out of these sample, 65(40 females, 25 males) (4.8\%) were tested positive.

The samples were recruited through three methods: FISH analysis for 22q11deletion was requested by an external medical professional or a clinical geneticist of the outpatient genetic clinic in 842 adults of whom 48(5.7 \%) were tested positive. Two hundred eighty six (286) adult subjects were tested at the genetic clinics organized in institutions for intellectually disabled and13 (4.5 \% ) were found to have the deletion. Thirdly, FISH analysis for 22q11 deletion was performed in113 adults seen as part of a general screening program in a 
psychiatric inpatient unit for intellectually disabled and out of this group 4 (3.5\%) had a 22q11 deletion.”

\section{Procedure}

A comprehensive study of the medical records was performed in all 65 patients and included data on psychiatric history and cognitive functioning. Only features assessed by a medical doctor (physical and psychiatric diagnosis) or a psychologist (cognitive level) were included.

A clinical genetic examination was performed by a clinical geneticist in all referred individuals and included data on pedigree, clinical examination, routine chromosome and FISH analysis. Earlier missed symptoms or signs were defined as symptoms or signs of 22q11DS that were not mentioned in the referral letter by the referring physician, but were found on the physical examination by the clinical geneticist. Presenting symptoms and signs and missed earlier symptoms and signs included diagnostic criteria for 22q11DS.

All 48 adults (17 males and 31 females) diagnosed at the genetic clinic were referred by a general practitioner or a medical specialist. Presenting symptoms were defined as the main symptoms or signs that lead to a referral. If there was more than one presenting symptom, the main presenting symptom was defined as the symptom that lead to the initial consultation with the referring specialist, for example if a cardiologist referred an adult because of a cardiac defect and intellectual disability, the cardiac defect was considered to be the main presenting symptom.

A similar procedure was used for adults diagnosed at the institutional genetic clinics but FISH analysis for 22q11 deletion was performed only in those residents who, in addition to the known intellectual disability, met one or more of the 6 other broad categories of clinical screening criteria for 22q11DS. Those include facial anomalies, hypernasal speech, cardiac 
defect, thymic hypoplasia, hypocalcemia or other birth defects [11]. Psychiatric disorders or severe behavior problems were considered as an additional category and a criterion to perform FISH analysis. Thirteen individuals (20\%) (4 males and 9 females) were diagnosed at the institutional genetic clinics. Intellectual disability was considered to be the main presenting feature while the other features leading to the decision to perform FISH analysis were considered to be a secondary or tertiary features.

Four adults (3 males and one female) were diagnosed as part of a general screening program for del 22q11 in adults admitted to a psychiatric unit for intellectually disabled. Informed consent was obtained from the patients and/or their parents or legal guardian. In this group, FISH analysis for detection of 22q11 deletion was performed in all patients regardless of the phenotypic features.

Age at diagnosis was defined as the age at which the result of the FISH analysis was available.

Presenting symptoms were categorized in seven groups based on the classification of McDonald-McGinn and Sullivan [1] as described in the introduction: familial occurrence, intellectual disability, cardiac anomalies, palatal anomalies, facial dysmorphic features, psychiatric problems and 'other' (comprising all other features associated with 22q11DS). Intellectual capacities were assessed using the Wechsler scales. If no IQ data were available, an estimation of daily functioning was made based on data about early psychomotor development, learning difficulties at school age and level of professional functioning in adulthood. Intellectual disability was classified as borderline IQ (FSIQ 70-85 or a developmental age of 12 year), mild intellectual disability (ID) (FSIQ 55-70 or a developmental age of 4 to 12 years), moderate ID (FSIQ 40-55 or a developmental age of two to 4 years), severe ID (IQ 25-40 or a developmental age of one to two years) or profound ID (FSIQ $<25$ or a developmental age of less than one year) [12]. 


\section{Results}

\section{Age at diagnosis}

The mean age at diagnosis (table 1) in the total sample was 34.03 years (range 18 - 60). The average age at diagnosis for individuals diagnosed at the genetic clinic was 30.9 years (range 18-54), while the average age at diagnosis for residents of the institutional clinic was 39.4 years (range 24 - 60). Patients diagnosed at the psychiatric unit for intellectually disabled were diagnosed at an average age of 51.5 years (range 45-58).

\section{Table 1}

Distribution of the study group and age at diagnosis according to the source of ascertainment

\begin{tabular}{lll}
\hline & Number (male/female) & $\begin{array}{l}\text { Mean age at diagnosis in } \\
\text { years (range) }\end{array}$ \\
\hline $\begin{array}{l}\text { Outpatient genetic clinic } \\
\text { Institution for }\end{array}$ & $13(4 / 9)$ & $30.3(18-54)$ \\
$\begin{array}{l}\text { intellectually disabled } \\
\begin{array}{l}\text { Psychiatric unit for } \\
\text { patients with dual } \\
\text { diagnosis }\end{array}\end{array}$ & $4(3 / 1)$ & $41(24-60)$ \\
\hline
\end{tabular}

\section{Presenting symptoms}

\section{Outpatient genetic clinic $(n=48)$}

Familial occurrence $(n=22)$

Twenty two adults (45.8\%) were referred to the clinic (table 2) because of a newly diagnosed family member. Only seven out of these 22 adults (31.4\%) presented with additional symptoms : three persons had craniofacial abnormalities including one diagnosed with TF, one with a history of learning difficulties and one working in a sheltered workshop. The secondary presenting symptom in the fourth patient was a VSD. Two adults were 
diagnosed with mild intellectual disability and one adult had a history of several psychiatric admissions because of paranoid schizophrenia. Mean age at diagnosis was 32.4 years (range 18-47 years)

\section{Table 2}

Overview of the main presenting symptoms and age at diagnosis in adults referred to the genetic clinic

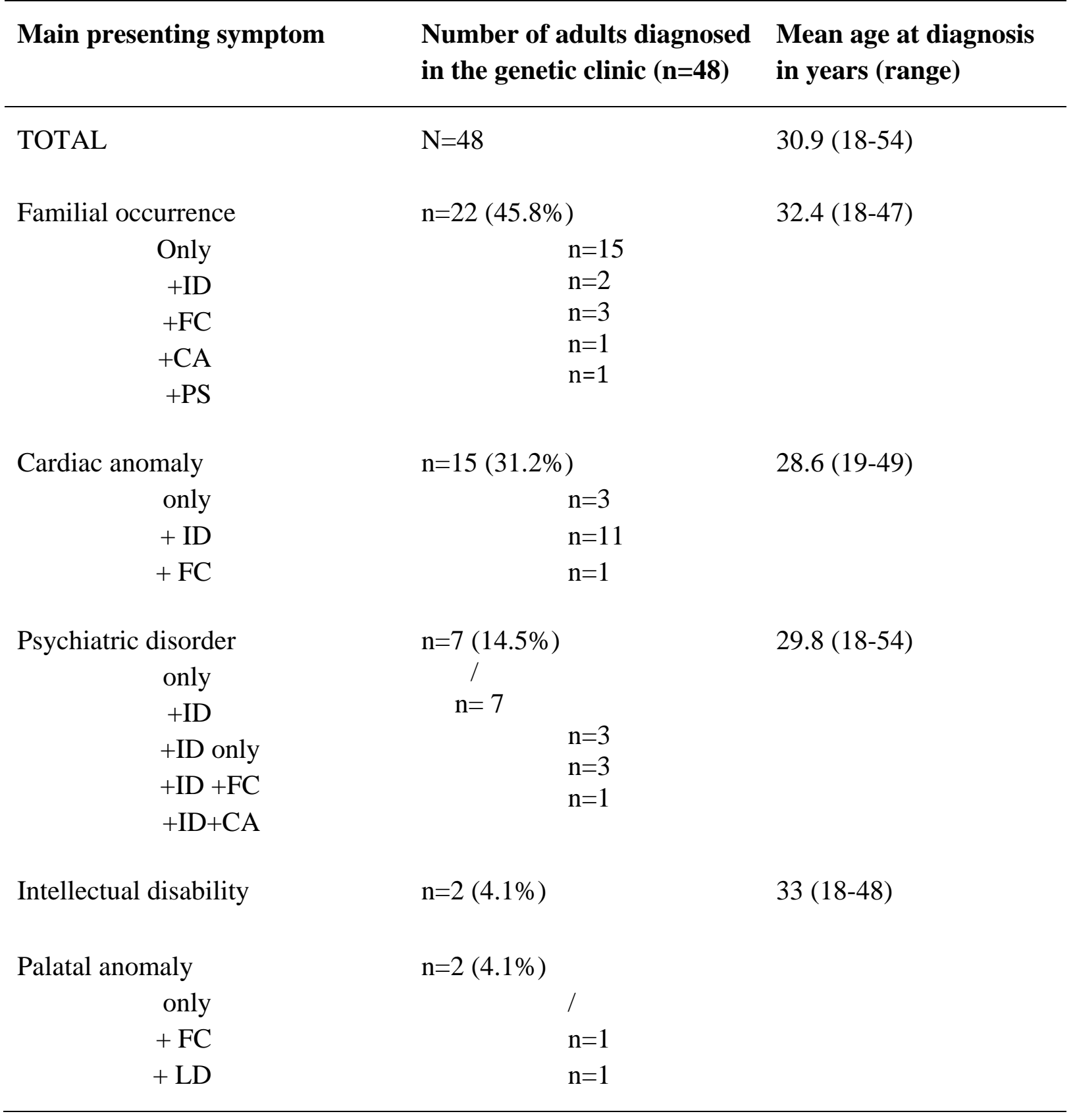


ID: intellectual disability; FC: facial characteristics; CA: cardiac anomalies; LD: learning difficulties; PS: psychiatric disorders

These individuals were internally referred by a geneticist after the initial 22q11DS diagnosis in the relative $(\mathrm{n}=18)$ or they were referred by the gynecologist or the pediatrician after the 22q11DS diagnosis in the fetus or newborn $(n=4)$.

Twenty one of them had a child with 22q11DS. In one adult the diagnosis was made after her sister and then her mother were shown to carry the del22q11.2.

\section{Cardiac anomalies $(n=15)$}

A cardiac problem was the main presenting symptom in 15 adults (31.2\%) and in three (3/15) of them it was the only presenting symptom. These three adults presented with different cardiac malformations: one had tetralogy of Fallot (TF), another one was diagnosed with Ventricle Septal Defect (VSD) with myocard hypertrophy and the third one was referred because of TF with pulmonary atresia and major aortopulmonary collateral artery.

The other 12 adults(12/15) were referred because of a cardiac problem associated with secondary symptoms such as intellectual disability $(n=11)$ orcraniofacial abnormalities $(n=1)$. Of those presenting with a cardiac malformation and intellectual disability, 7 had also tertiary presenting symptoms including craniofacial abnormalities $(n=4)$, short stature and delay of puberty $(n=1)$, epilepsy $(n=1)$ and renal agenesis $(n=1)$. The person with craniofacial abnormalities as a secondary symptom had learning difficulties as a tertiary symptom. Out of these 12 adults, 9 were diagnosed with conotruncal malformations with tetralogy of Fallot (TF) in all of them. Five adults presented with TF only while the other four had a complicated form of TF: TF with double-outlet right ventricle (DORV) in combination with VSD (n=1), TF withDOVR , type agenesis pulmonary valve( $n=1)$, TF with a right turning aortic arch $(\mathrm{n}=1)$ and TF with truncus arteriosus $(\mathrm{n}=1)$. Three of these 12 adults presented with non- 
conotruncal heart defects: atrium septal defect $(n=1)$, atrioeptal defect in combination with VSD and coarctation of the aortic arch ( $\mathrm{n}=1)$ and AVSD (n=1).

Mean age at diagnosis was 28.6 years (range 19-49 years). Four adults with a cardiac defect died (age at death: 31, 38, 44, 58 years).

Eleven out of 15 individuals were referred by a (pediatric) cardiologist while two adults were referred by a pediatrician. One adult was referred by a general practitioner. Referral data were lacking in one adult with cardiac anomalies and intellectual disability.

Psychiatric problems $(n=7)$

Seven patients were referred because of a psychiatric disorder or severe behavioural problem in combination with intellectual disability. In all of them the combination of psychiatric disorder and borderline intelligence or mild intellectual disability were the reason for referral and considered as equally important. One intellectually disabled psychiatric patient was referred with an additional cardiac anomaly (TF). Three intellectually disabled adults had craniofacial characteristics including facial dysmorphy and nasal speech. Two adults presented with anxieties including one with a comorbid psychiatric diagnosis of autism spectrum disorder. Two adults suffered from psychosis in combination with depression and two were diagnosed with schizophrenia. Behavioral problems with verbal and physical aggression was reported in one person.

Mean age at diagnosis was 29.8 years (range 18 to 54 years).

Five patients including the adult with TF were referred by the psychiatrist. The other two adults were referred by the general practitioner. 
Intellectual disability $(n=2)$

Two adults were referred to the genetic clinic with intellectual disability as a primary presenting symptom and craniofacial dysmorphism with velopharyngeal insufficiency as a secondary symptom. Out of these two , one individual presented with severe and chronic thrombocytopenia as a tertiary symptom. Both individuals were referred by general practitioners with a special interest in intellectual disability. Mean age at diagnosis was 30.6 years (18 and 48 years).

Twelve individuals presented with two or more features whereby intellectual disability was considered to be a secondary feature helpful in the decision to refer to the genetic clinic. The main presenting symptoms in these adults were: cardiac problems $(n=7)$ and psychiatric disorders $(n=5)$.

Palatal anomalies $(n=2)$

A palatal anomaly was the only presenting problem in two adults, both referred by the otorhinolaryngologist. Mean age at diagnosis was 22 years (19 and 23 years).

\section{Facial features}

None of the adults were referred because of their craniofacial phenotype. Facial features were noticed and explicitly mentioned by the referring cardiologist in one subject with cardiac anomalies and intellectual disability.

None of the patients were referred because of problems of immune deficiency, hypocalcaemia or a history of feeding problems. 


\section{Missed symptoms}

Hypernasal speech with a history of feeding problems, and facial dysmorphy (micrognathia and posterior rotated ears) were the most commonly missed earlier symptoms of 22q11DS and were noted in 18 (40\%) and 17 (37.7\%) individuals respectively. A mild degree of intellectual disability had not been reported as a presenting symptom in 13 (28.8 \%) adults. Cardiac problems ( $\mathrm{n}=2$ or $4.4 \%$ ) and psychiatric disorders ( $\mathrm{n}=2$ or $4.4 \%$ ) were not mentioned as presenting symptoms by the referring professional in 4 subjects. Other missed symptoms ( $n=16$ or $35.5 \%$ ) included scoliosis, a history of recurrent infections, hearing loss and (a history of) thrombocytopenia.

\section{Institutions for intellectually disabled $(n=13)$}

Data on intelligence quotient were available in seven adults and the mean IQ was 61 with a range from 46 to 85 . Out of the 13 institutionalized adults, one had borderline intelligence (FSIQ= 85), two had a mild intellectual disability (FSIQ = 62 and 66), one had a moderate intelligence (FSIQ 46). An estimation of cognitive functioning was available in two adults: one with moderate and one with severe intellectual disability. Cognitive data were missing in the other three adults.

Cardiac anomalies $(n=3)$

Three intellectually disabled adults presented with a cardiac anomaly including one with TF and two with VSD of whom one also had pulmonary hypertension. The two adults with VSD also had a history of psychiatric problems but the exact diagnosis was not known (Table 3) 
Psychiatric problems $(n=6)$

Six adults had a history of psychiatric disorder in addition to the intellectual disability. Five were diagnosed with a psychotic disorder and one adult was described with a schizophrenic personality. Comorbid psychiatric diagnoses included autism, depression, anxieties and obsessive compulsive disorder.

Facial features $(n=3)$

Craniofacial dysmorphy was found in three intellectually disabled adults including one who had nasal speech.

Other $(n=1)$

One intellectually disabled female presented with thrombocytopenia. She had a history of hypothyroidism, anxieties, depression with suicidal ideation and hallucinations.

\section{Table 3}

Overview of the main presenting symptoms in adults seen at the genetic clinic in institutions for intellectually disabled. ID: intellectual disability

\section{Main (ID) and secondary Institution for intellectually presenting symptoms disabled $(n=13)$}

TOTAL

ID and cardiac anomaly

ID and psychiatric disorder

ID and facial features only +palatal anomaly

$$
\mathrm{n}=13
$$$$
\mathrm{n}=3(23 \%)
$$$$
\mathrm{n}=6(46 \%)
$$

$$
\begin{aligned}
& \mathrm{n}=3(23 \%) \\
& \mathrm{n}=2(10 \%) \\
& \mathrm{n}=1(10 \%)
\end{aligned}
$$




\section{Psychiatric inpatient unit for adults with intellectual disability $(n=4)$}

All four adults were intellectually disabled and were diagnosed with a psychiatric disorder. Three adults had a moderate intellectual disability (mean FSIQ: 54, range 49-60) while one had a mild intellectual disability (FSIQ 60). One adult was diagnosed with bipolar disorder while a second one presented with psychotic disorder paranoid type. Two adults presented with severe physical and verbal aggression.

\section{Discussion}

Even if made late during adulthood, the diagnosis of 22q11DS remains of crucial importance because of the wide range of medical problems that may occur, resulting in a diminished life expectancy, and increased risk of sudden death [13].

In this study, out of the total 22q11DS population diagnosed after the age of 18 years, nearly three fourth were referred to the outpatient genetic clinic and one fourth was actively recruited.

The mean ages of diagnosis in the actively recruited groups in the institutions and in the psychiatric hospital (respectively 41.0 en 51.7 years) were higher than the mean age at diagnosis in adults referred to the genetic clinic (30.3 years). The earlier age at diagnosis in adults seen at the outpatient clinic is mainly the result of familial occurrence i.e. nearly half of the adults were diagnosed as young parents following the diagnosis of an affected child. One third of the adults referred after an earlier diagnosis in a family member presented with additional cardiac, cognitive or psychiatric features. This is contradictory to a previous study of adults identified through an affected relative [14], where all 18 adults had only relatively mild clinical manifestations and were diagnosed following an earlier diagnosis in an affected child. The author pointed out that the presence of minimal clinical signs in 22q11DS parents 
with an affected child [14], suggest that the adults who survive to reproduce are those without major medical complications of the disorder [5].. Our study indicates that also parents with moderate to severe clinical manifestations such as psychiatric disorders and cardiac defects may remain undiagnosed until they have an affected child.

In this study, one third of the adults referred to the genetic clinic presented with conotruncal heart defects, mainly TOF. Nearly all of them were referred by a cardiologist of the same hospital reflecting the close collaboration of the genetic clinic and the clinic for cardiology. This high prevalence of cardiac anomalies in adults corresponds to the data of a previous study that reported cardiac anomalies in one fourth of the adults [15].

This high rate of cardiac anomalies in adults referred to the genetic clinic is in contrast with the absence of cardiac anomalies in the adults diagnosed in the institutions for intellectually disabled or diagnosed in the psychiatric unit for intellectually disabled. This low prevalence of cardiac defects in the institutionalized adults of our study may be explained by a higher neonatal mortality before 1980. All institutionalized and hospitalized adults but one were 39 years or older and thus born well before major advances in cardiosurgery. They belong to a generation with low survival of congenital heart defect (CHD. Another explanation for the low prevalence of CHD in the older institutionalized and hospitalized intellectually disabled adults, may be the elevated risk of premature death in adults with 22q11DS. [13].

Although palatal anomalies were considered as a reason for referral in only two individuals, it was noticed at the time of the clinical examination by the geneticist in nearly half of the adults. This prevalence rate is consistent with the data of a previous study were palatal anomalies including submucous cleft and velopharyngeal insufficiency were present in 
$42 \%$ of the adults [15]. These data may indicate that palatal anomalies and/or velopharyngeal insufficiency in adults are easily overlooked. [17].

Psychiatric disorders including psychotic disorders, anxiety disorders and mood disorders are well described in adults with 22q11DS, occur in up to 60\% [18] and are considered to be a prominent problem in adulthood [8]. The psychiatric illness of most concern in the literature is psychotic disorder observed in up to one third of the adults $[19,20]$. Most psychotic disorders emerge during the late teens, twenties or thirties and one might expect that a relatively high number of patients with mild physical manifestations are diagnosed on the occasion of one of these first psychotic episodes. In this study only 14\% (7/48) presented with psychiatric problems and only 4.\% (2/48) presented with a psychotic disorder. This low number of adults presenting with psychotic disorders in our total population confirms the previous theory of Kook et al. [21] that 22q11DS is rarely suspected among adults presenting with a psychotic disorder but without physical abnormalities. On the other hand, we included all adults older than 18 and there is still a risk that some of them might develop a psychiatric disorder.

Most individuals (50\%) with 22q11DS have borderline intelligence, about 30-40\% have mild intellectual disability and nearly all of them have a history of learning difficulties at school [15, 22]. Although we did not systematically perform a formal assessment of intelligence, most adults referred to the genetic clinic had an intellectual disability ranging from mild ID to borderline intelligence.

The majority of the institutionalized adults have a moderate or severe intellectual disability compared to the borderline intelligence or mild intellectual disability in those referred to the genetic clinic. As there is evidence for a high vulnerability for mental disorder in moderate to severely intellectually disabled individuals [23, 24] one may expect institutionalised 22q11DS 
adults present more often with psychotic disorders. We found that $46 \%$ of the institutionalised population were diagnosed with 22q11DS because of a history of psychotic disorders.

Comorbidity was high with autism, depression, anxieties, and obsessive compulsive disorder. It is possible that those with a history of psychotic disorders experience a general decline in intelligence and adaptive functioning leading to placement in institutions for intellectually disabled. Indeed, several studies found that a decrease in verbal skills accompanies the onset of psychotic disorders in 22q11DS [25-28]. Another explanation may be that clinical geneticists working at these institutional clinics are familiar with the psychiatric phenotype of this syndrome and have an enhanced index of suspicion which may contribute to a higher detection rate.

Moderate or severe levels of intellectual disability are known to be rare in 22q11DS [15, 30] and the diagnosis is rarely considered in this population. It is therefore possible that without the active recruitment policy of the genetic clinic, the adults residing in institutions for intellectually disabled and in the psychiatric unit for intellectually disabled would never have been diagnosed.

Although the clinical geneticist noticed typical craniofacial features in nearly half of the adults only one physician mentioned these features in the referral letter. This is consistent with a previous report [17] stating that the facial features are not as distinctive or abnormal as in some other genetic syndromes and thus may be easily missed by non-geneticists. Clinical geneticists are used to evaluate facial features and one might thus expect a high detection rate in the genetic clinics for institutionalized intellectually disabled adults but only a few patients showed the typical craniofacial phenotype. The average age of the population seen at the institutions is high and it may be possible that the typical facial features become less evident with age. Facial features may also be less pronounced in adults with a more severe form of 
intellectual disability and/or a long history of psychotic disorders and use of psychopharmaceuticals.

We thus found that adults with a moderate to severely intellectually disability are diagnosed at an older age, rarely have less typical craniofacial features, have a high risk for psychiatric disorders and a lower risk for cardiac malformations. These findings are confirmed by the results of a general screening for del22q11 in a population of adults with moderate to severe intellectual disability and severe psychiatric disorders. Out of the 113 adults tested, four were tested positive for 22q11DS and none of them showed, apart from the intellectual disability any of the clinical screening criteria for 22q11DS. All four of them were older than 45 at the time of diagnosis and the chance that they would have been diagnosed outside this screening program is low.

These results raise the possibility that the deletion in moderate to severely intellectually disabled adults with psychiatric disorders and without any physical symptoms is more common than previously reported and easily missed. It emphasizes the need to broaden the index of suspicion for developmental psychiatrists.

\section{Conclusion}

Familial occurrence is the most frequent indication for adults with 22q11DS to be referred to the genetic clinic followed by cardiac anomalies with or without other features. A moderate to severe developmental delay with a psychiatric disorder were the main presenting symptoms in adults ascertained through intellectually disabled resources. This emphasis the need to stay alert for presenting symptoms such as moderate to severe intellectual disability in combination with a history of psychiatric disorders, particularly in the absence of obvious physical features. 
In summary, an increased index of suspicion in moderately to severely retarded adults with severe psychiatric disorders as well as in adults with a borderline intelligence and cardiac defects and/or a history of feeding problems and hypernasal speech could lead to more diagnoses of 22q11DS and benefit the patient and his family.

\section{References}

[1] McDonald-McGinn DM, Sullivan KE. Chromosome 22q11.2 deletion syndrome (DiGeorge syndrome/velocardiofacial syndrome). Medicine 2011; 90: 1-18.

[2] Bassett AS, McDonald-McGinn DM, Devriendt K, Digilio MC, Goldenberg P, Habel A, Marino B, Oskarsdottir S, Philip N, Sullivan K., Swillen A, Vorstman J. Practical guidelines for managing patients with 22q11.2 deletion syndrome. J Peds. 2011; 159(2): $332-9$.

[3] Gothelf D, Frisch A, Michaelovsky E, Weizman A, Shprintzen RJ. Velo-cardio-facial syndrome. J Ment Health Res Intellect Disabil. 2009; 2(2): 149-67.

[4] Robin NH, Shprintzen RJ. Defining the clinical spectrum of deletion 22q11.2. J Pediatr. 2005; 147: 90-6.

[5] Swillen A, Vogels A, Devriendt K, Fryns JP. Chromosome 22q11 deletion syndrome: update and review of the clinical features, cognitive-behavioral spectrum, and psychiatric complications. Am J Med Genet. (Semin Med Genet.) 2000; 97: 128-35.

[6] Ballesta Martínez MJ, Guillén Navarro E, López Expósito I, Baffaliu Vidal JA, Domingo Jiménez R, Guía Torrent JM, Robles Sánchez F, Sánchez Solís de Querol M. Review of 22 patients with 22q11.2 deletion syndrome; phenotype spectrum. An Pediatr. (Barc.) 2008; 69(4): 304-10.

[7] Momma K. Cardiovascular anomalies associated with chromosome 22q11.2 deletion syndrome. Am J Cardiol. 2010; 102(11): 1617-24. 
[8] Lima K, Følling I, Eiklid KL, Natvig S, Abrahamsen TG. Age-dependent clinical problems in a Norwegian national survey of patients with the 22q11.2 deletion syndrome. Aur J Pediatr. 2010; 169(8): 983-9.

[9] Óskardóttir S, Persson C, Eriksson BO, Fasth A. Presenting phenotype in 100 children with the 22q11 deletion syndrome. Eur J Pediatr. 2005; 164: 146-53.

[10] Costain G, Chow EW, Ray PN, Bassett AS. Caregiver and adult patient perspectives on the importance of a diagnosis of 22q11.2 deletion syndrome. J Intellect Disabil Res. 2012; 56(6): 641-51.

[11] Basset AS, Chow EW. 22q11 deletion syndrome: a genetic subtype of schizophrenia. Biol Psychiatry 1999; 46: 882-91.

[12] World Health Organization. ICD (International Classification of Diseases and related Health Problems). Geneva, Switzerland: WHO; 2010.

[13] Bassett AS, Chow EW, Husted J, Hodgkinson KA, Harris L, Silversides C. Premature death in adults with 22q11.2 deletion syndrome. J Med Genet. 2009; 46(5): 324-30.

[14] McDonald-McGinn DM, Tonnesen MK, Laufer-Cahana A, Finucane B, Driscoll DA, Emanuel BS, Zackai EH. Phenotype of the 22q11.2 deletion in individuals identified through an affected relative: cast a wide FISHing net! Genet Med. 2001; 3(1): 23-9.

[15] Bassett AS, Chow EWC, Husted J, Weksberg R, Caluseriu O, Webb GD, Gatzoulis MA. Clinical features of 78 adults with 22q11 deletion syndrome. Am J Med Genet. 2005; 138A: 307-13.

[16] Agergaard P, Hebert A, Sørensen KM, Østergaard JR, Olesen C. Can clinical assessment detect 22q11.2 deletions in patients with cardiac malformations? A review. Eur J Med Genet. 2011; 54(1): 3-8.

[17] Shprintzen RJ. Velo-cardio-facial syndrome: 30 years of study. Dev Disabil Res Rev. 2008; 14: 3-10. 
[18] Fung WLA, McEvilly R, Fong J, Silversides C, Chow E, Bassett A. Elevated prevalence of generalized anxiety disorder in adults with 22q11.2 deletion syndrome. Am J Psychiatry 2010; 167(8): 998.

[19] Gothelf D, Gruber R, Presburger G, Dotan I, Brand-Gothelf A, Burg M, Inbar D, Steinberg T, Frisch A, Apter A, Weizman A. . Methylphenidate treatment for attentiondeficit/hyperactivity disorder in children and adolescents with velocardiofacial syndrome: an open-label study. J Clin Psychiatry 2003; 64: 1163-9.

[20] Bassett AS, Chow EWC, AbdelMalik P, Gheorghiu M, Husted J, Weksberg R. The schizophrenia phenotype in 22q11 deletion syndrome. Am J Psychiatry 2003; 160(9): 1580-6.

[21] Kook SD, An SK, Kim KR, Kim WJ, Lee E, Namkoong K. Psychotic features as the first manifestation of 22q11.2 deletion syndrome. Psychiatry Investig. 2010; 7(1): 72-4.

[22] De Smedt B, Devriendt K, Fryns JP, Vogels A, Gewillig M, Swillen A. Intellectual abilities in a large sample of children with 22Q11DS: an update. J Intellect Disabil Res. 2007; 51: 666-70.

[23] Fletcher R, Loschen E, Stavrakaki C, First M. Diagnostic Manual-Intellectual Disability (DM-ID): a textbook of diagnosis of mental disorders in persons with intellectual disability. Kingston, NY: NAPP Press (National Association for the Dually Diagnosed); 2007.

[24] Nettelbladt P, Goth M, Bogren M, Mattisson C. Risk of mental disorders in subjects with intellectual disability in the Lundby cohort 1947-97, Nord J Psychiatry 2009; 63: 316-21.

[25] Gothelf D, Eliez S, Thompson T, Hinard C, Penniman L, Feinstein C, Kwon H, Jin S, Jo B.; Antonarakis SE, Morris MA, Reiss AL.. COMT genotype predicts longitudinal 
cognitive decline and psychosis in 22q11.2 deletion syndrome. Nat Neurosci. 2005; 8(11): 1500-2.

[26] Green T, Gothelf D, Glaser B, Debbane M, Frisch A, Kotler M, Weizman A, Eliez S. Psychiatric disorders and intellectual functioning throughout development in velocardiofacial (22q11.2 deletion) syndrome. J Am Acad Child Adolesc Psychiatry. 2009; 48(11): 1060-1.

[27] Antshel KM, Shprintzen R, Fremont W, Higgins AM, Faraone SV, Kates WR. Cognitive and psychiatric predictors to psychosis in velocardiofacial syndrome: a 3-year follow-up study. J Am Acad Child Adolesc Psychiatry 2010; 49(4): 333-44.

[28] Philip N, Bassett A. Cognitive, behavioural and psychiatric phenotype in 22q11.2 deletion syndrome. Behav Genet. 2011; 41(3): 403-12.

[29] Chow EWC, Watson M, Young DA, Bassett AS. Neurocognitive profile in 22q11 deletion syndrome and schizophrenia. Schizophr Res. 2006; 87(1-3): 270-8. 